\title{
A Possible Generalization of Acoustic Wave Equation Using the Concept of Perturbed Derivative Order
}

\author{
Abdon Atangana ${ }^{1}$ and Adem Kilıçman ${ }^{2}$ \\ ${ }^{1}$ Institute for Groundwater Studies, Faculty of Natural and Agricultural Sciences, University of the Free State, \\ Bloemfontein 9300, South Africa \\ ${ }^{2}$ Department of Mathematics and Institute for Mathematical Research, University Putra Malaysia, 43400 Serdang, Malaysia
}

Correspondence should be addressed to Adem Kılıçman; akilicman@putra.upm.edu.my

Received 18 February 2013; Accepted 18 March 2013

Academic Editor: Guo-Cheng Wu

Copyright (C) 2013 A. Atangana and A. Kılıçman. This is an open access article distributed under the Creative Commons Attribution License, which permits unrestricted use, distribution, and reproduction in any medium, provided the original work is properly cited.

\begin{abstract}
The standard version of acoustic wave equation is modified using the concept of the generalized Riemann-Liouville fractional order derivative. Some properties of the generalized Riemann-Liouville fractional derivative approximation are presented. Some theorems are generalized. The modified equation is approximately solved by using the variational iteration method and the Green function technique. The numerical simulation of solution of the modified equation gives a better prediction than the standard one.
\end{abstract}

\section{Introduction}

Acoustics was in the beginning the study of small pressure waves in air which can be detected by the human ear: sound. The possibility of acoustics has been extended to higher and lower frequencies: ultrasound and infrasound. Structural vibrations are now often included in acoustics. Also the perception of sound is an area of acoustical research. In our present paper we will limit ourselves to the original definition and to the propagation in fluids like air and water. In such a case acoustics is a part of fluid dynamics. A major problem of fluid dynamics is that the equations of motion are nonlinear. This implies that an exact general solution of these equations is not available. Acoustics is a first-order approximation in which nonlinear effects are neglected. The corresponding relative density fluctuations $\rho^{\prime} / \rho_{0}$ are considered very small [1]. The acoustic wave equation governs the propagation of acoustic waves through a material medium. The form of the equation is a second-order partial differential equation. The equation describes the evolution of acoustic pressure $P$ or particle velocity $u$ as a function of position $r$ and time $t$. A simplified form of the equation that describes acoustic waves in only one spatial dimension is considered in this paper

$$
\frac{\partial^{2} P}{\partial x^{2}}-\frac{1}{c^{2}} \frac{\partial^{2} P}{\partial t^{2}}=0
$$

A derivation of general linearized wave equations is discussed by Pierce and Goldstein $[1,2]$. However, neglecting the nonlinear effects in this equation, may lead to inaccurate prediction of the propagation of acoustic wave through the medium. Therefore in order to include explicitly the effect of corresponding relative density fluctuations in the mathematical formulation, one needs to insert it in the partial differential equation that governs the propagation of the acoustic wave. Recently, the acoustic equation was extended to the concept of fractional order derivative in [3]. Therefore in this paper, our concern is the modification of the previous equation by perturbing the order of the first derivative by replacing the first order of the derivative with $1+\varepsilon$ where $\varepsilon$ is a positive small parameter. Also, when we consider diffusion process in porous medium, if the medium structure or external field changes with time, in this situation, the ordinary integer order and constant-order fractional diffusion equation model cannot be used to well characterize such phenomenon (see [3-9]).

\section{Definitions and Approximation}

To describe the propagation of acoustic waves through a material medium with coordinate and time-dependent perturbed dimension, one must use Riemann-Liouville 
fractional order derivative that was introduced and used in a number of works (see $[3,6-8,10])$. These derivatives are defined as (see $[3,6-8]$ )

$$
\begin{aligned}
D^{1+\varepsilon_{t}} & =D_{+, t}^{\mu_{t}} f \\
& =\left(\frac{d}{d t}\right)^{n} \int_{0}^{t}\left[\frac{f(\tau)}{\Gamma\left(n-\mu_{\tau}(\tau)(t-\tau)^{\mu_{\tau}-n+1}\right)}\right] d \tau, \\
D^{1+\varepsilon_{x}} & =D_{+, x}^{\mu_{x}} f \\
& =\left(\frac{d}{d x}\right)^{n} \int_{0}^{x}\left[\frac{f(\tau)}{\Gamma\left(n-\mu_{\tau}(\tau)(t-\tau)^{\mu_{\tau}-n+1}\right)}\right] d \tau .
\end{aligned}
$$

Here, $\Gamma$ is the Euler gamma function; $n=\{\mu\}+1$, where $\{\mu\}$ is the integer part of $\mu$ for $\mu \geq 0$, that is, $n-1 \leq \mu<n$ and $n=0$ for $\mu<n$. Following (2) we have that $\mu_{t}=1+\varepsilon_{t}$ and $\mu_{x}=1+\varepsilon_{x}$. The integral operator defined previously for fractional exponents $\mu_{x}$ and $\mu_{t}$ depending on coordinates and time can be expressed in terms of ordinary derivative and integral [11] for $|\varepsilon| \ll 1$. Here $\varepsilon_{t}$ and $\varepsilon_{x}$ are considered as the corresponding relative density fluctuations $\rho^{\prime} / \rho_{0}$ that vary slightly in time and space, respectively. For this matter, generalized Riemann-Liouville fractional derivatives satisfy the approximate relations:

$$
\begin{aligned}
& D^{1+\varepsilon_{t}} f \cong\left(1+\varepsilon_{t}\right) \frac{\partial f}{\partial t}+\frac{\partial \varepsilon_{t}}{\partial t} f \\
& D^{1+\varepsilon_{x}} f \cong\left(1+\varepsilon_{x}\right) \frac{\partial f}{\partial x}+\frac{\partial \varepsilon_{x}}{\partial x} f .
\end{aligned}
$$

The previous relations make it possible to describe the dynamic system, including the effect of the corresponding relative density fluctuations, by means of partial differential and integral equations.

\section{Some Properties of the Approximation}

Let us examine some properties of the previous derivative operator.

(i) Addition

If $u_{x}, f(x)$, and $g(x)$ are differentiable in the opened interval I then,

$$
D^{1+u_{x}}[f(x)+g(x)] \cong D^{1+u_{x}}[f(x)]+D^{1+u_{x}}[g(x)] .
$$

Proof. We have

$$
\begin{aligned}
D^{1+u_{x}} & {[f(x)+g(x)] } \\
\cong & \left(1+u_{x}\right) \frac{\partial[f(x)+g(x)]}{\partial x} \\
& +\frac{\partial u_{x}}{\partial x}[f(x)+g(x)]\left(1+u_{x}\right) \frac{\partial[f(x)]}{\partial x} \\
& +\frac{\partial u_{x}}{\partial x}[f(x)]+\left(1+u_{x}\right) \frac{\partial[g(x)]}{\partial x}+\frac{\partial u_{x}}{\partial x}[g(x)] \\
\cong & D^{1+u_{x}}[f(x)]+D^{1+u_{x}}[f(x)] .
\end{aligned}
$$

\section{(ii) Division}

If $u_{x}$ and $1 / f(x)$ are differentiable on the open interval I then

$$
\begin{aligned}
D^{1+u_{x}}\left[\frac{1}{f(x)}\right] & \cong \frac{\left[-\left(1+u_{x}\right) f^{\prime}(x)+u_{x}^{\prime} f(x)\right]}{f^{2}(x)} \\
& =\frac{-f^{\prime}(x)}{f^{2}(x)}-\frac{u_{x} f^{\prime}(x)}{f^{2}(x)}+\frac{u_{x}^{\prime} f(x)}{f^{2}(x)} .
\end{aligned}
$$

I then

(iii) Multiplication

If $u_{x}, f(x)$ and $g(x)$ are differentiable in the open interval

$$
\begin{aligned}
D^{1+u_{x}}[f(x) \cdot g(x)] \cong & g(x) f^{\prime}(x)+f(x) g^{\prime}(x) \\
& +\left(g f^{\prime}+f g^{\prime}\right)(x) u_{x} \\
& +u_{x}^{\prime}(f(x) g(x)) .
\end{aligned}
$$

(iv) Power

If $u_{x}$ and $f(x)$ are differentiable in the open interval I then

$$
\begin{aligned}
D^{1+u_{x}}\left[(f(x))^{n}\right] \cong & n f^{\prime} f^{n-1} \\
& +u_{x} n f^{\prime} f^{n-1}+u_{x}^{\prime} f^{n}, \quad n \geq 1 .
\end{aligned}
$$

If $u_{x}$ and $f(x)$ are two times differentiable in the open interval I then

$$
\begin{aligned}
& D^{1+u_{x}}\left[D^{1+u_{x}}[f(x)]\right] \cong\left(1+u_{x}\right) \\
& \times\left[\left(1+u_{x}\right) \frac{\partial^{2} f}{\partial x^{2}}+3 \frac{\partial f}{\partial x} \frac{\partial u_{x}}{\partial x}+\frac{\partial^{2} u_{x}}{\partial x^{2}} f\right]+\frac{\partial u_{x}}{\partial x} f .
\end{aligned}
$$

3.1. Clairaut's Theorem for the Approximation. Assume that $f(x, y), u_{x}$, and $u_{y}$ are functions for which $\partial^{2} f / \partial x \partial y$, $\partial^{2} f / \partial y \partial x, \partial^{2} \varepsilon_{x} / \partial x \partial y$, and $\partial^{2} \varepsilon_{y} / \partial x \partial y$ exist and are continuous over a domain $D \subset \mathbb{R}^{2}$ then, $D^{1+u_{x}}\left[D^{1+u_{y}}[f(x, y)]\right]$ and $D^{1+u_{y}}\left[D^{1+u_{x}}[f(x, y)]\right]$ exist and are continuous over the domain D. If in addition $u_{x}=u_{y}$ then

$$
\begin{aligned}
D^{1+u_{y}} & {\left[D^{1+u_{x}}[f(x, y)]\right] } \\
& =D^{1+u_{x}}\left[D^{1+u_{y}}[f(x, y)]\right] .
\end{aligned}
$$

Proof. If $f(x, y), u_{x}$, and $u_{y}$ are functions for which $\partial^{2} f / \partial x \partial y, \partial^{2} f / \partial y \partial x, \partial^{2} \varepsilon_{x} / \partial x \partial y$, and $\partial^{2} \varepsilon_{y} / \partial x \partial y$ exist and are continuous over a domain $D \subset \mathbb{R}^{2}$ then

$$
\begin{aligned}
& D^{1+u_{y}}\left[D^{1+u_{x}}[f(x, y)]\right] \\
& \cong\left(1+u_{y}\right)\left[\frac{\partial u_{x}}{\partial y} \frac{\partial f}{\partial x}+\frac{\partial^{2} f}{\partial y \partial x}+\frac{\partial^{2} u_{x}}{\partial y \partial x} f(x, y)+\frac{\partial u_{x}}{\partial x} \frac{\partial f}{\partial y}\right] \\
& \quad+\left(1+u_{x}\right) \frac{\partial u_{y}}{\partial y} \frac{\partial f}{\partial x}+\frac{\partial u_{y}}{\partial y} \frac{\partial u_{x}}{\partial x} f(x, y) .
\end{aligned}
$$

Now interchanging $x$ by $y$ we obtain $D^{1+u_{x}}\left[D^{1+u_{y}}[f(x, y)]\right]$. 
If $u_{x}=u_{y}$ then $\partial^{2} f / \partial y \partial x=\partial^{2} f / \partial x \partial y$ according to Clairaut's theorem; thus replacing $\partial^{2} f / \partial x \partial y$ by $\partial^{2} f / \partial y \partial x$ in $D^{1+u_{y}}\left[D^{1+u_{x}}[f(x, y)]\right]$, we obtain that

$$
D^{1+u_{y}}\left[D^{1+u_{x}}[f(x, y)]\right]=D^{1+u_{x}}\left[D^{1+u_{y}}[f(x, y)]\right] .
$$

\subsection{Chain-Rule for the Approximation. We have}

$$
\begin{aligned}
D^{1+u_{x}}(f \circ g) \cong & \left(1+u_{x}\right) g^{\prime}(x) f^{\prime}[g(x)]+\frac{\partial \varepsilon_{x}}{\partial x}(f \circ g) \\
= & g^{\prime}(x) f^{\prime}[g(x)]+u_{x} g^{\prime}(x) f^{\prime}[g(x)] \\
& +\frac{\partial \varepsilon_{x}}{\partial x}(f \circ g) .
\end{aligned}
$$

3.3. Rolle's Theorem for the Approximation. If a real-valued functions $f$ and $u_{x}$ are continuous on a closed interval $[a, b]$, differentiable on the open interval $(a, b)$, and $f(a)=f(b)$, then there exist a $c$ in the open interval $(a, b)$ and a small parameter $\mu$ such that

$$
D^{1+u_{x}} f(c)=\mu f(c) .
$$

Proof. Following Rolle's theorem, there exists a $c$ in the open interval $(a, b)$ such that $f^{\prime}(c)=0$. For this $c$ we have that

$$
\begin{aligned}
D^{1+u_{x}} f(c) & =\left(1+u_{x}(c)\right) f^{\prime}(c)+u_{x}^{\prime}(c) f(c) \\
& =u_{x}^{\prime}(c) f(c) \\
& =\mu f(c) .
\end{aligned}
$$

If $g(x), f(x)$, and $u_{x}$ are differentiable in an open interval I, then there exist $2>\alpha>1$ and $\beta>0$ such that

$$
\begin{aligned}
\left|D^{1+u_{x}} f(x)-D^{1+u_{x}} g(x)\right| & \leq \alpha\left|f^{\prime}(x)-g^{\prime}(x)\right| \\
& +\beta|f(x)-g(x)|, \quad \forall x \in I .
\end{aligned}
$$

Proof. Let $x \in I$; then

$$
\begin{aligned}
&\left|D^{1+u_{x}} f(x)-D^{1+u_{x}} g(x)\right| \\
&=\mid \mid\left(1+u_{x}(x)\right) f^{\prime}(x)+u_{x}^{\prime}(x) f(x) \\
& \quad-\left(1+u_{x}(x)\right) g^{\prime}(x)-u_{x}^{\prime}(x) g(x) \mid \\
&=\left|1+u_{x}(x)\right|\left|f^{\prime}(x)-g^{\prime}(x)\right|+\left|u_{x}^{\prime}(x)\right||f(x)-g(x)| .
\end{aligned}
$$

But $u_{x}(x)$ is very small such that $\left|1+u_{x}(x)\right|<2$ and $\left|u_{x}^{\prime}(x)\right|>$ 0 ; it follows that

$$
\begin{aligned}
& \left|1+u_{x}(x)\right|\left|f^{\prime}(x)-g^{\prime}(x)\right|+\left|u_{x}^{\prime}(x)\right||f(x)-g(x)| \\
& \leq \alpha\left|f^{\prime}(x)-g^{\prime}(x)\right|+\beta|f(x)-g(x)| .
\end{aligned}
$$

It is important to observe that if $u=0$, we recover the properties of normal derivatives.

\section{Modification of the Equation}

In order to include explicitly the possible effect of the corresponding relative density fluctuations into the mathematical formulation, in this paper, we replace the classical version of the derivative of (1) by the modified Riemann-Liouville fractional derivative approximation (3) to obtain

$$
D^{1+\varepsilon_{x}}\left[D^{1+\varepsilon_{x}} P\right]=\frac{1}{c^{2}} D^{1+\varepsilon_{t}}\left[D^{1+\varepsilon_{t}} P\right] .
$$

Making use of (3) and relation (8), the previous equation can be transformed to the following partial differential equation for $\varepsilon \ll 1$ :

$$
\begin{gathered}
\left(1+\varepsilon_{x}\right)\left[\left(1+\varepsilon_{x}\right) \frac{\partial^{2} P}{\partial x^{2}}+3 \frac{\partial P}{\partial x} \frac{\partial \varepsilon_{x}}{\partial x}+\frac{\partial^{2} \varepsilon_{x}}{\partial x^{2}} P\right]+\frac{\partial \varepsilon_{x}}{\partial x} P \\
=\frac{1}{c^{2}}\left\{( 1 + \varepsilon _ { t } ) \left[\left(1+\varepsilon_{t}\right) \frac{\partial^{2} P}{\partial t^{2}}\right.\right. \\
\left.\left.+3 \frac{\partial P}{\partial t} \frac{\partial \varepsilon_{t}}{\partial t}+\frac{\partial^{2} \varepsilon_{t}}{\partial t^{2}} P\right]+\frac{\partial \varepsilon_{t}}{\partial t} P\right\} .
\end{gathered}
$$

Omitting the terms of $\varepsilon^{2}$ in the previous equation, we obtain the following:

$$
\begin{aligned}
& \left(1+2 \varepsilon_{x}\right) \frac{\partial^{2} P}{\partial x^{2}}+3 \frac{\partial P}{\partial x} \frac{\partial \varepsilon_{x}}{\partial x}+\frac{\partial^{2} \varepsilon_{x}}{\partial x^{2}} P \\
& =\frac{1}{c^{2}}\left\{\left(1+2 \varepsilon_{t}\right) \frac{\partial^{2} P}{\partial t^{2}}+3 \frac{\partial P}{\partial t} \frac{\partial \varepsilon_{t}}{\partial t}+\frac{\partial^{2} \varepsilon_{t}}{\partial t^{2}} P\right\} .
\end{aligned}
$$

Now since the small parameters representing the perturbation additions to unity are small, the right- and left-hand sides of (21) can be divided by $1+2 \varepsilon_{x}$. In this case, we obtain

$$
c(x, t) \frac{\partial^{2} P}{\partial t^{2}}=\frac{\partial^{2} P}{\partial x^{2}}+F(x, t)+B(x, t) P .
$$

Here, We have,

$$
\begin{aligned}
& c(x, t)=\frac{1}{c^{2}}\left(1+2 \varepsilon_{t}-2 \varepsilon_{x}\right), \\
& F(x, t)=3\left(\frac{\partial P}{\partial x} \frac{\partial \varepsilon_{x}}{\partial x}-\frac{1}{c^{2}} \frac{\partial P}{\partial t} \frac{\partial \varepsilon_{t}}{\partial t}\right), \\
& B(x, t)=\left(\frac{\partial^{2} \varepsilon_{x}}{\partial x^{2}}-\frac{1}{c^{2}} \frac{\partial^{2} \varepsilon_{t}}{\partial t^{2}}\right) .
\end{aligned}
$$

It is easy to observe that (22) differs from (1) in three properties.

First the velocity of the sound in this case depends on time and coordinates due to the effect of the corresponding relative density fluctuations. Secondly the force

$$
F(x, t)=3\left(\frac{\partial P}{\partial x} \frac{\partial \varepsilon_{x}}{\partial x}-\frac{1}{c^{2}} \frac{\partial P}{\partial t} \frac{\partial \varepsilon_{t}}{\partial t}\right)
$$

appears due to the coordinate and time dependence of the corresponding relative density fluctuations, and such 
force was considered in [12]. Third there is a derivativefree term that depends on both time and space, and it is proportional to $P$ and characterizes, depending on the coefficient sign, the retardation or enhancement propagation of acoustic waves through a material medium. Therefore, even weak memory, which is taken into account by generalized Riemann-Liouville fractional derivatives and presents the characteristics of a corresponding relative density fluctuations, transforms constant-coefficient velocity to varyingcoefficient velocity. Moreover, this memory is responsible for a force with which the corresponding relative density fluctuations act on a propagation of acoustic waves through a material medium. This force appears only if the propagating acoustic wave has memory depending on coordinates and time; that it "remembers" its trajectories and time. Those terms in (22) that involve the corresponding relative density fluctuations additions $(F$ and $B$ ) to the time and space dimensions are very small. Also the exact analytical solution of this modified equation is not easy to be determined. Therefore, this equation can be solved approximately by changing the function $P(r, t)$ to $P_{0}(r, t)$, which satisfies (1).

\section{Solutions of the Modified Equation}

The modified equation can be reformulated as follows:

$$
-\frac{1}{c^{2}} \frac{\partial^{2} P}{\partial t^{2}}+\frac{\partial^{2} P}{\partial x^{2}}=H\left(\varepsilon_{x}, \varepsilon_{t}, P_{0}(r, t)\right) .
$$

To solve (25) we need to give explicitly $\varepsilon_{x}$ and $\varepsilon_{t}$. For example if one consider these functions to be

$$
\varepsilon_{x}=\varepsilon_{o x} \sin \theta_{x} x, \quad \varepsilon_{t}=\varepsilon_{o t} \sin \theta_{t} t,
$$

where $\varepsilon_{o x}$ and $\varepsilon_{o t}$ are very significantly small such that $|\varepsilon| \ll 1$. Since these parameters are very small then the solution of (25) can be sought in the form $P=P_{1}+P_{0}$ where $P_{0}$ is the solution of (1) and $P_{1} \ll P_{0}$ is proportional to $\varepsilon_{x}$ and $\varepsilon_{t}$. Here $\theta_{x}$ and $\theta_{t}$ are frequencies characterizing variation in corresponding relative density fluctuations.

To solve (25) together with (26) we make use of two techniques including the Green function techniques and the variational iterative decomposition technique. Here we will start with the variational iteration techniques.

5.1. Variational Iteration Method. Variational iteration method has been favourably applied to various kinds of nonlinear problems. The main property of the method is in its flexibility and ability to solve nonlinear equations accurately and conveniently. Very recently it was recognized that the variational iteration method [11, 13-20] can be an effective procedure for solution of various nonlinear problems without usual restrictive assumptions. In this paper we will make use of this iterative decomposition technique to solve the modified wave (26) together with (25). To solve (25) by means of variational iteration method, we put (25) in the form of

$$
\left(P_{1}(x, t)\right)_{2 x}-\frac{1}{c^{2}}\left(P_{1}(x, t)\right)_{2 t}-H(x, t)=0 .
$$

The correction functional for (5) can be approximately expressed for this matter as follows:

$$
\begin{aligned}
P_{n+1}(x, t)= & P_{n}(x, t) \\
& -\int_{0}^{x} \lambda(\zeta)\left[\frac{\partial^{m} P(\zeta, t)}{\partial \zeta^{m}}-\frac{1}{c^{2}}(\widetilde{P(\zeta, t)})_{2 t}-\widetilde{H(\zeta, t)}\right] d \zeta,
\end{aligned}
$$

where $\lambda$ is a general Lagrange multiplier [21,22], which can be recognized optimally by means of variation assumption [21, 22]; here $\left({\widetilde{P(\zeta, t))_{2 t}}}_{\text {, and }}\right.$, $\widetilde{H(r, \tau)}$ are considered as constrained variations. Making the previous functional stationary, we obtain

$$
\begin{aligned}
\delta P_{n+1}(x, t)= & \delta P_{n}(x, t) \\
& -\delta \int_{0}^{x} \lambda(\zeta)\left[\frac{\partial^{m} P(\zeta, t)}{\partial \zeta^{m}}\right] d \zeta .
\end{aligned}
$$

Capitulates the next Lagrange multipliers, giving up to the following Lagrange multipliers $\lambda=-1$ for the case where $m=$ 1 and $\lambda=x-\zeta$ for $m=2$. For these matters $m=2$, we obtain the following iteration formula:

$$
\begin{aligned}
P_{n+1}(x, t)= & P_{n}(x, t)-\int_{0}^{x}(x-\zeta) \\
& \times\left[\frac{\partial^{2} P_{n}(\zeta, t)}{\partial \zeta^{2}}-\frac{1}{c^{2}} \frac{\partial^{2} P_{n}(\zeta, t)}{\partial t^{2}}-H(\zeta, t)\right] d \zeta .
\end{aligned}
$$

It is worth noting that if the zeroth component $P_{0}(x, t)$ is defined, then the remaining components $n \geq 1$ can be completely determined such that each term is determined by using the previous terms, and the series solutions are thus entirely determined. Finally, the solution $P(r, t)$ is approximated by the truncated series

$$
\begin{gathered}
P_{N}(x, t)=\sum_{n=0}^{N-1} P_{n}(x, t), \\
\lim _{N \rightarrow \infty} P_{N}(r, t)=P_{1}(x, t) .
\end{gathered}
$$

Here we choose the first term to be zero meaning $P_{0}(x, t)=P(0, t)=0$ and the second term can be determined as

$$
P_{1}(x, t)=-\int_{0}^{x}(x-\zeta)[-H(\zeta, t)] d \zeta .
$$

Our next concern is to define $H(x, t)$; that is, we first need to provide the solution of (1) which is found in the literatures [23]. The following solutions are obtained by separation of variables in different coordinate systems. They are phasor solutions, meaning that they have an implicit timedependence factor of $\exp (i \omega t)$ where $\omega=2 \pi f$ is the angular frequency. The explicit time dependence is given by (33)

$$
P(x, k, t)=\operatorname{Real}[p(k, x) \exp [i \omega t]], \quad k=\frac{\omega}{c} .
$$




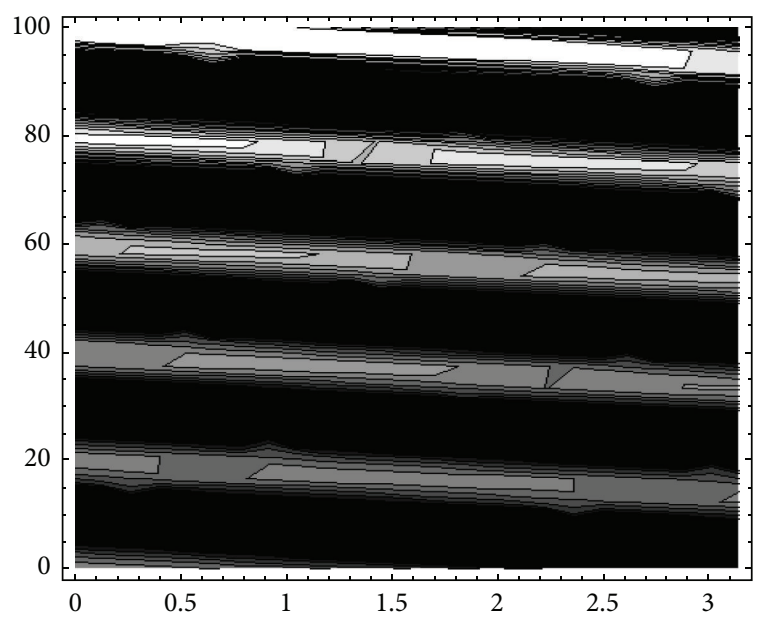

FIgURE 1: Topographic map of the solution of the modified acoustic equation for $c=4, f=0.001$.

Introducing the previous expression in $H(x, t)$, we obtain the second expression. In this matter two components of the decomposition series were obtained of which $P(x, t)$ was evaluated to have the following expansion:

$$
P_{1}(x, t)=P_{1}(x, t)+P_{0}(x, t)+\cdots
$$

The next figures show the graphical representation of the approximated solution of the modified acoustic wave equation and the exact solution of the standard version of acoustic wave equation as function of space and time (contour plot and density plot of both solution). A contour plot gives essentially a topographic map of a solution and the density plot shows the values of the function at a regular erray of points and lighter region of the contour plot is higher.

From the next Figures 1, 2, 3, and 4, respectively, one can see that there are more details with the solution of the modified acoustic wave equation than in the standard solution, meaning that the details left out by neglecting the small effect of the correspondent relative density fluctuations are very important when one needs to observe the propagation of the acoustic wave through the material medium.The approximate solution of (25) has been depicted in Figures 1 and 3 and the exact solution in Figures 2 and 4.

5.2. Green Function Method. To solve (25) together with (26) by means of Green function technique, one needs first to construct a suitable green function.

If $G$ is the green function to be constructed, then $G$ must satisfy the following equation:

$$
\begin{gathered}
\frac{\partial^{2} G\left(x, t \mid x_{0}, t_{0}\right)}{\partial x^{2}}-\frac{1}{c^{2}} \frac{\partial^{2} G\left(x, t \mid x_{0}, t_{0}\right)}{\partial t^{2}} \\
=-4 \pi \delta\left(x-x_{0}\right) \delta\left(t-t_{0}\right) .
\end{gathered}
$$

We are lucky enough, because the Green function to be constructed here is the green function of the wave equation

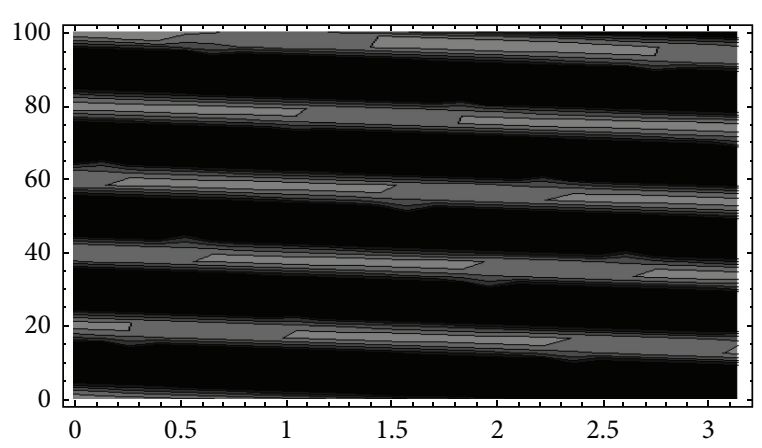

FIGURE 2: Topographic map of solution of acoustic wave equation for $c=4$ and $f=0.001$.

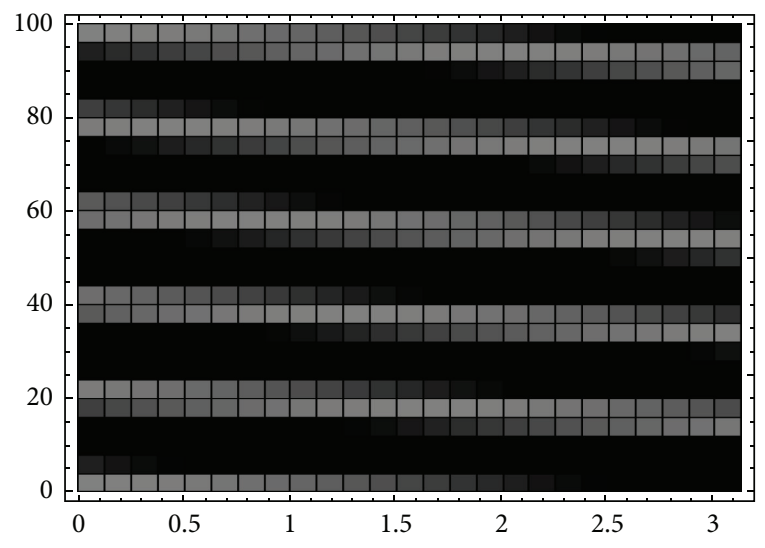

FIgURE 3: Density plot of the solution of the modified acoustic wave equation.

and is given later in the case of the closed forms for the Green function for the infinite one-dimensional domain [23]:

$$
G\left(x, t \mid x_{0}, t_{0}\right)=2 \pi c u\left(\left(t-t_{0}\right)-\left(\left|x-x_{0}\right|\right)\right),
$$

where

$$
u(x)=0 \quad \text { if } x<0, \quad u(x)=1 \quad \text { if } x>0 .
$$

Following the Green function technique, the general solution of the modified acoustic wave equation is given later as

$$
P_{1}(x, t)=\int_{0}^{t} \int_{0}^{x} G\left(x_{1}, t_{1} \mid x_{0}, t_{0}\right) H\left(x_{1}, t_{1}\right) d t_{1} d x_{1},
$$

where $H\left(x_{1}, t_{1}\right)$ remains the same as defined earlier in Section 5.1.

\section{Conclusion}

In this paper, an acoustic wave equation was extended to the concept of the modified Riemann-Liouville fractional order derivative. We presented in detail some properties of the generalized Riemann-Liouville fractional order derivative approximation. We presented the analysis of the generalized equation. We highlighted the three differences between the 


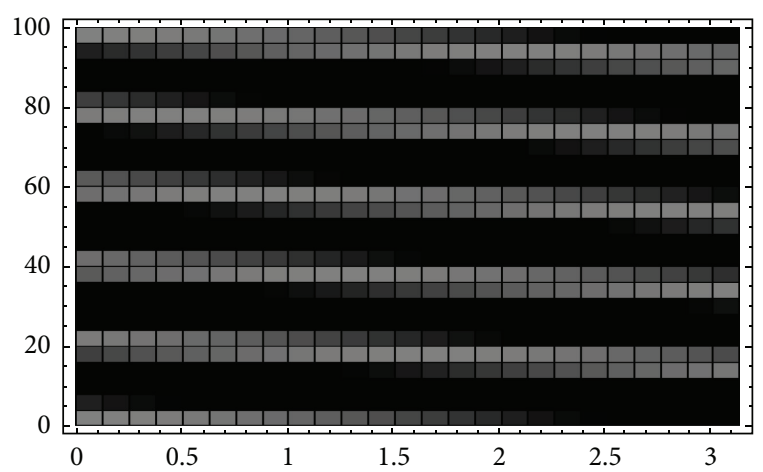

FIGURE 4: Density plot of the solution of the acoustic wave equation.

generalized equation and the standard one. First the velocity of the sound in this case depends on time and coordinates due to the effect of the corresponding relative density fluctuations. Second the force appears due to the coordinate and time dependence of the corresponding relative density fluctuations. Third there is a derivative-free term that depends on both time and space which is proportional to $P$ and characterizes, depending on the coefficient sign, the retardation or enhancement propagation of acoustic waves through a material medium. The modified equation is approximately solved by using the variational iteration method and the Green function technique. The solution of the modified equation gives better prediction than the standard one.

\section{References}

[1] K. Aki and P. Richards, Quantitative Seismology, Theory and Methods: Freeman, New York, NY, USA, 1980.

[2] A. D. Pierce, Acoustics: An Introduction to Its Physical Principles and Applications, McGraw-Hill Book Company, New York, NY, USA, 1981.

[3] W. Chen and S. Holm, "Fractional Laplacian time-space models for linear and nonlinear lossy media exhibiting arbitrary frequency dependency," Journal of the Acoustical Society of America, vol. 115, no. 4, pp. 1424-1430, 2004.

[4] L. E. S. Ramirez and C. F. M. Coimbra, "On the selection and meaning of variable order operators for dynamic modeling," International Journal of Differential Equations, vol. 2010, Article ID 846107, 16 pages, 2010.

[5] L. E. S. Ramirez and C. F. M. Coimbra, "On the variable order dynamics of the nonlinear wake caused by a sedimenting particle," Physica D, vol. 240, no. 13, pp. 1111-1118, 2011.

[6] B. Ross and S. Samko, "Fractional integration operator of variable order in the Hölder spaces $H^{\lambda(x)}$," International Journal of Mathematics and Mathematical Sciences, vol. 18, no. 4, pp. 777-788, 1995.

[7] S. Umarov and S. Steinberg, "Variable order differential equations with piecewise constant order-function and diffusion with changing modes," Zeitschrift für Analysis und ihre Anwendungen, vol. 28, no. 4, pp. 431-450, 2009.

[8] R. L. Magin, O. Abdullah, D. Baleanu, and X. J. Zhou, "Anomalous diffusion expressed through fractional order differential operators in the Bloch-Torrey equation," Journal of Magnetic Resonance, vol. 190, no. 2, pp. 255-270, 2008.
[9] A. Atangana and J. F. Botha, "Generalized groundwater flow equation using the concept of variable order derivative," Boundary Value Problems, vol. 2013, p. 53, 2013.

[10] H. G. Sun, W. Chen, and Y. Q. Chen, "Variable order fractional differential operators in anomalous diffusion modeling," Physica A, vol. 388, no. 21, pp. 4586-4592, 2009.

[11] G. C. Wu, "New trends in the variational iteration method," Communications in Fractional Calculus, vol. 2, no. 2, pp. 59-75, 2011.

[12] L. Ya. Kobelev, L. Ya. Kobelev, and L. Yu. Klimontovicht" "Anomalous diffusion with time and coordinate-dependent memory," Dokiady Physics, vol. 48, no. 6, pp. 264-268, 2003.

[13] G. C. Wu and D. Baleanu, "Variational iteration method for the Burgers' flow with fractional derivatives-New Lagrange multipliers," Applied Mathematical Modelling, vol. 37, no. 9, pp. 6183-6190, 2013.

[14] G. C. Wu and D. Baleanu, "Variational iteration method for fractional calculus-a universal approach by Laplace transform," Advances in Difference Equations, vol. 2013, p. 18, 2013.

[15] S. A. El-Wakil, E. M. Abulwafa, M. A. Zahran, and A. A. Mahmoud, "Time-fractional KdV equation: formulation and solution using variational methods," Nonlinear Dynamics, vol. 65, no. 1-2, pp. 55-63, 2011.

[16] A. Atangana, A. Ahmed, and N. Bildik, "A generalized version of a low velocity impact between a rigid sphere and a transversely isotropic strain-hardening plate supported by a rigid substrate using the concept of non-integer derivatives," Abstract Applied Analysis, vol. 2013, Article ID 671321, 9 pages, 2013.

[17] A. Atangana and A. Secer, "Time-fractional coupled- the korteweg-de vries equations," Abstract Applied Analysis, vol. 2013, Article ID 947986, 8 pages, 2013.

[18] J. J. S. Duan, R. Rach, D. Bulean, and A. M. Wazwaz, "A review of the Adomian decomposition method and its applications to fractional differential equations," Communications in Fractional Calculus, vol. 3, no. 2, pp. 73-99, 2012.

[19] N. Bildik and A. Konuralp, "The use of variational iteration method, differential transform method and Adomian decomposition method for solving different types of nonlinear partial differential equations," International Journal of Nonlinear Sciences and Numerical Simulation, vol. 7, no. 1, pp. 65-70, 2006.

[20] Z. M. Odibat and S. Momani, "Application of variational iteration method to nonlinear differential equations of fractional order," International Journal of Nonlinear Sciences and Numerical Simulation, vol. 7, no. 1, pp. 27-34, 2006.

[21] M. Inokuti, H. Sekine, and T. Mura, "General use of the Lagrange multiplier in non-linear mathematical physics," in Variational Method in the Mechanics of Solids, S. Nemat-Nasser, Ed., pp. 156-162, Pergamon Press, Oxford, UK, 1978.

[22] A. Atangana, "New class of boundary value problems," Information Science Letters, vol. 1, no. 2, pp. 67-76, 2012.

[23] P. M. Morse and H. Feshbash, Methods of Theoretical Physics, McGraw-Hill, New York, NY, USA, 1953. 


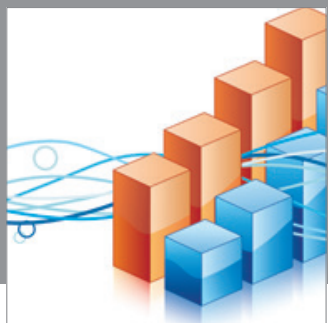

Advances in

Operations Research

mansans

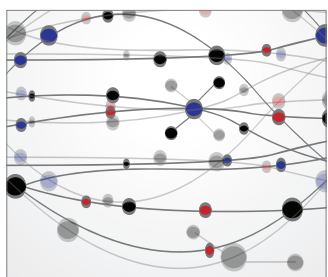

The Scientific World Journal
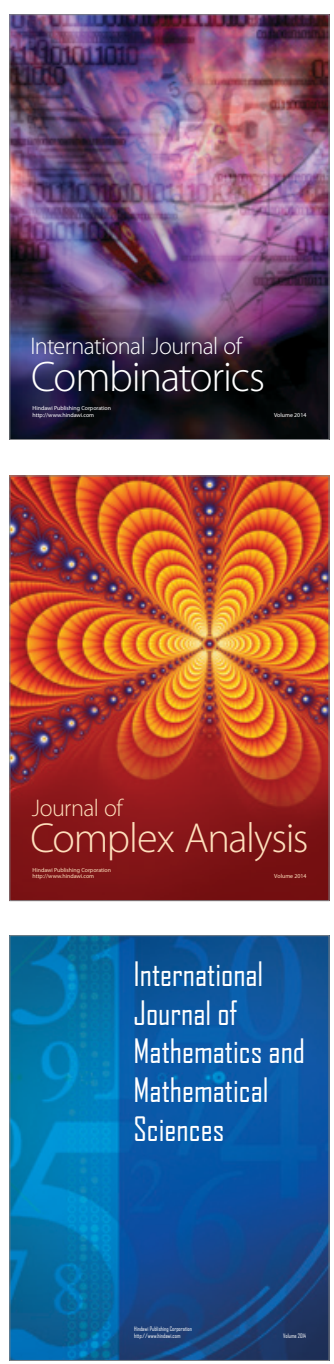
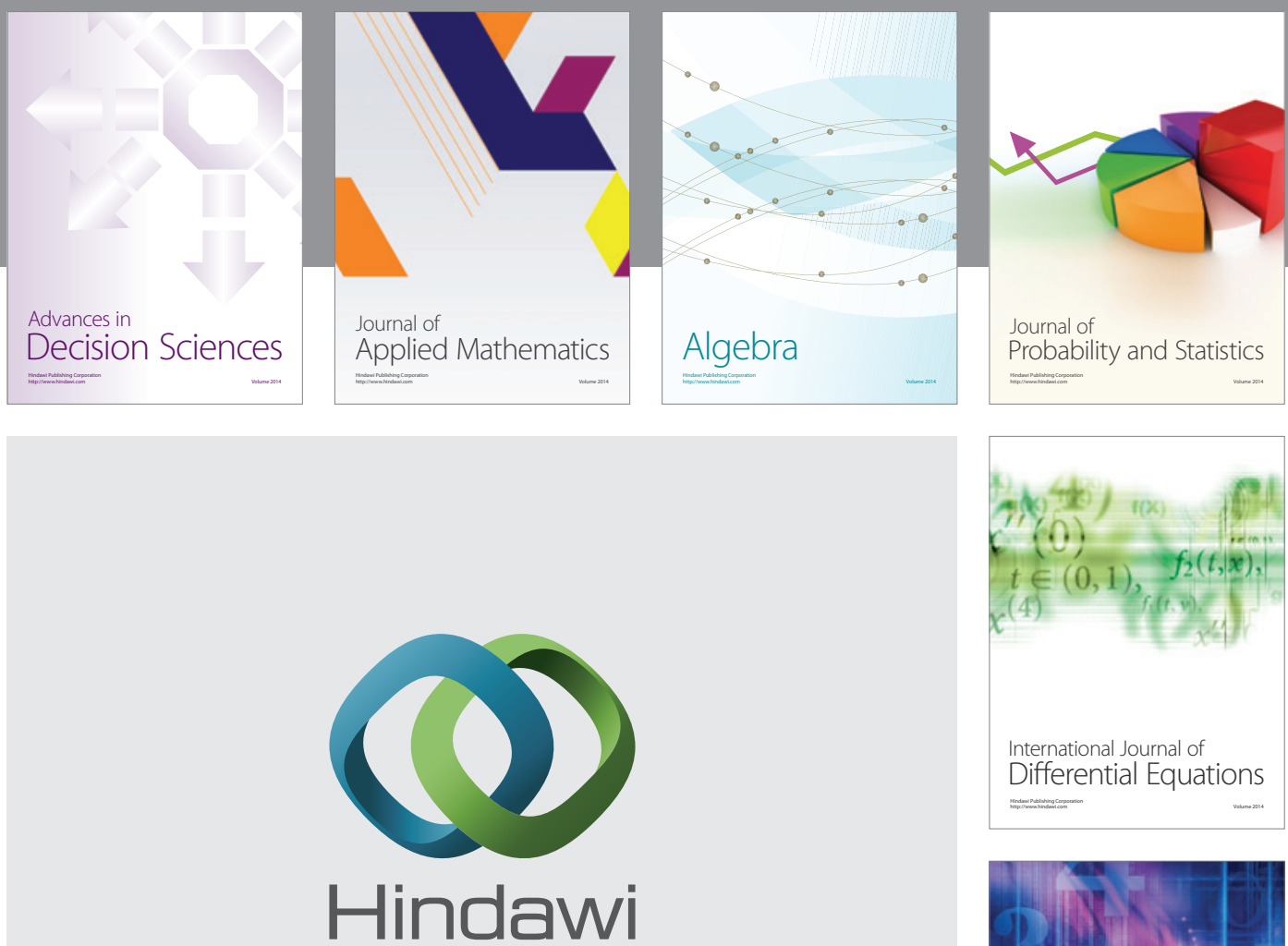

Submit your manuscripts at http://www.hindawi.com
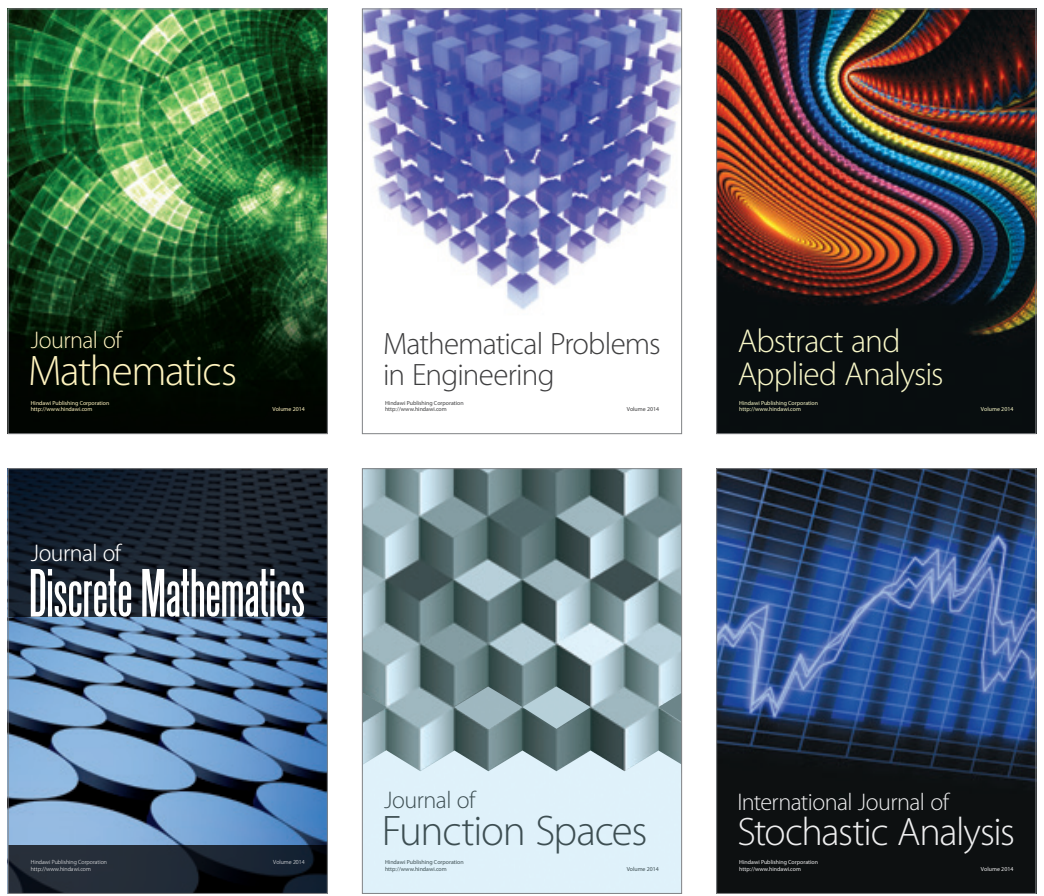

Journal of

Function Spaces

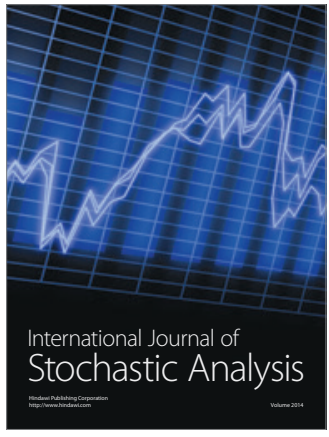

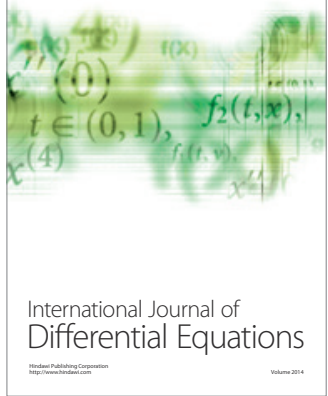
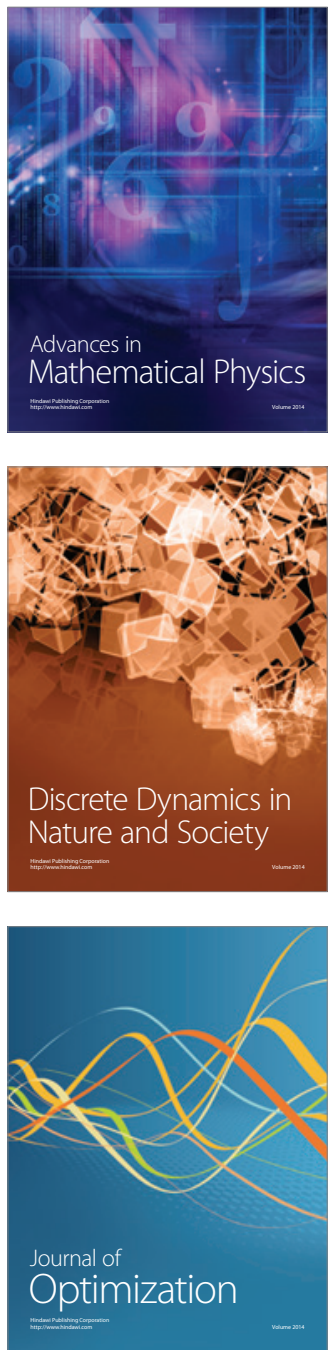